\title{
A Method to Determine System Parameters of Sdof Structural Systems by Applying a Step Load
}

\author{
Wenlung Li , and Kuo-Ching Liu \\ Dept. of Mechanical Engineering, National Taipei University of Technology, Taipei 10608, TAIWAN
}

\begin{abstract}
A novel method for identifying the physical parameters of structural systems has been proposed, unlike most existing methods are in terms of modal properties. Furthermore, the present method can be applied when structures are in services. The method is first theoretically derived by using single degree-of-freedom (sdof) systems, and will be generalized to multi-dof of lumped mass ones later. The present method requires a step external excitation, but the final expressions are independent of its input. Thus, it can be classified as an "output-only" method. In fact, it is the amplitudes of decaying spectrum, which is obtained by the so-called moving window Fourier transform (mwFT) from system signals is concerned, in addition to the steady-state deflection. Using the amplitude of this steady-state deflection, which can be obtained from the spectrum of the mwFT, as the quantity of calibration, the system parameters can be simultaneously estimated. The method is numerically verified by a model of bridge cranes.
\end{abstract}

\section{Introduction}

The so-called "inverse problems" are to identify the system parameters from the measured response signals or the outputs. This is quite important in engineering practices since the results can be used to monitor the health of a system. Basically, to accomplish this goal is to retrieve the dynamic properties from the available vibration signals which may include outputs along or both of inputs and outputs. Thus, one calls this group of engineering problem as a parameter identification problem as well.

The health of a system may directly in terms of to its physical properties, or indirectly to its modal parameters. The latter includes like the eigen-frequencies [1], mode shapes and damping ratios [2, 3], the former like energy, mass, damping and stiffness of the system. However, the two are well correlated, though one may more sensitive than the other in some cases. For example, low sensitivity in changes of natural frequencies and mode shapes has been observed [4] when relating degradation of system stiff-ness. Therefore, one intention of the present report is to derive an identification method that is directly applicable of the physical properties, instead of the modal ones.

In fact, the authors have provided an output-only algorithm to identify the damping coefficients of sdof and mdof systems. The method was later successfully applied to estimate the modal damping ratios of machine tools. However, it was found that the method is still limited in the modal properties. It was further generalized together with a known mass to a new one [5]. The advantages of the new method are notable. Readers are referred to that report. Yet, it is inconvenient in some applications like bridge cranes. Motivated by such application practices need, the authors thus prepare this report. In addition, all notations are kept unchanged as the former [5].

\section{Theoretical backgrounds}

\subsection{Basic concept}

Theoretically, the system response of an sdof structural system with mass $m$, damping $c$ and stiffness $k$ under a unit-step force is governed by

$$
m \ddot{x}+c \dot{x}+k x=f(t)
$$

where $f(t)$ is a step function with a rise time at $t_{0}=0$ and the final value $F_{0}$, or

$$
f(t)=F_{0} \cdot u\left(t-t_{0}\right)
$$

where $u(.$.$) is the unit-step function. The responses of eqn$ (1) can be represented as [e.g., 6]

$$
x(t)=x_{0}+e^{-\zeta \omega_{n} t}\left[C_{1}^{\prime} \cos \left(\omega_{d} t\right)+C_{2}^{\prime} \sin \left(\omega_{d} t\right)\right],
$$

where $x_{0}=F_{0} / k, \omega_{d}=\omega_{n} \sqrt{1-\zeta^{2}}$ is the damped natural circular frequency of the system, and

$$
\omega_{n}^{2}=k / m \quad \text { and } \quad \zeta=c /\left(2 m \omega_{n}\right),
$$

are the undamped natural circular frequency and the damping ratio of the system, respectively. Two constants in eqn (3), $C_{\text {.. }}^{\prime}$, can be determined from the initial conditions. For example, if both of initial conditions are 
zero, or $\dot{x}(0)=x(0)=0$, then the two constants are [e.g., $6]$

$$
C_{1}^{\prime}=-F_{0} / k \text { and } C_{2}^{\prime}=\frac{-F_{0} \zeta}{k \sqrt{1-\zeta^{2}}}
$$

respectively. For the reason of derivation completeness, however, the present report keeps them for now.

Depending on the type of sensors used, one is normally to measure either responses (or deflections) or accelerations if experimental measurements are carried out. Assuming that it is the structural deflections, $x(t)$, are of the main interest, one has the exponential form as

$$
x(t)=x_{0}+e^{-\zeta \omega_{n} t}\left[C_{1} e^{j \omega_{d} t}+C_{2} e^{-j \omega_{d} t}\right],
$$

and $C_{. .}$are the two complex constants, and $j=\sqrt{-1}$. Examining eqn (6) closely, there is no difficult to find that the system response can be treated as the linear combinations of the transient and steady-state responses. Nevertheless, applying the so-called moving window Fouier Transform (mwFT) to eqn (6), a windowed system response of eqn (6) is transformed by computing

$$
X(\Omega, \tau)=\int_{-\infty}^{\infty}[x(t) \cdot w(t-\tau)] \exp (-j \Omega t) d t
$$

where $w(.$.$) is the moving window function that located at$ $\tau$ in the time domain. That is, to virtually divide the transient signal $x(t)$, after discarded $L_{0}$, into several pieces by the window function, and individually take the FT for those windowed signals. Refer to the Parseval's power theorem [e.g., 6], the powers of the right-hand-side (RHS) and LHS of eqn (7) are equal within the individual windows. For example, if one considers a rectangular window centered at $t=\tau$, then the windowed $x(t)$ may be written as

$$
\begin{aligned}
x(t) \cdot w(t-\tau) & =x(t) . \\
& {[u(t-(\tau-L))-u(t-(\tau+L))], }
\end{aligned}
$$

where $L$ is the half-length of the window, cf. Figure 1. Note also that the length of the moving window $L$ can be a critical parameter. If $L$ is chosen too short, it may be not able to capture all essential temporal features. On the other hand, however, if $L$ is too long, it may include too much noise at the right tail into transformation in the real engineering practices. Nevertheless, one computes the power spectrum from eqn (7) by using Mathematica, and obtains

$$
\begin{aligned}
X(\Omega, \tau)=a_{0}+\frac{j}{\sqrt{2 \pi}} & {\left[C_{1} \frac{e^{-j(\tau-L) A_{1}}-e^{j(\tau+L) A_{1}}}{A_{1}}\right.} \\
& \left.+C_{2} \frac{e^{-j(\tau-L) A_{2}}-e^{j(\tau+L) A_{2}}}{A_{2}}\right]
\end{aligned}
$$

where $A_{1}$ and $A_{2}$ are both complex functions of $\omega_{d}$ and defined by

$$
A_{1,2}(\Omega)=\left(\Omega \pm \omega_{d}\right)+j\left(\zeta \omega_{n}\right) .
$$

Notice also that the first term in RHS of eqn (9), $a_{0}$, is the mean of the oscillating windowed signal, cf. Figure 2. It is also real, time-invariant and the Fourier coefficient if one further compares eqn (9) with (3). Or, it can be read from any one of the power spectra of the windowed signals. Thus, it is no difficult to write

$$
a_{0}=x_{0}=\frac{F_{0}}{k},
$$

from the average of $x(t)$ and the corresponding spectrum. That is, the stiffness is related to the mean $\left(a_{0}\right.$ or $\left.\mathrm{dc}\right)$ of a power spectrum. Note that the accuracy of $x_{0}$ is vital to the whole identification since it has been chosen as the calibration quantity. Care should be taken when to identifying $a_{0}$. Figure 1 graphically shows the concept to estimate $a_{0}$ of the Fourier coefficient.

On the other hand, if one restricts the damping ratio is reasonably small ( $\zeta \ll 1.0$, says $10 \%$ for example) by examining eqn (9), then only the first term in the brackets governs the result when imposing $\Omega \rightarrow \omega_{d}$. The reason stems form that the denominator of this term is much smaller than the other, while the numerators are in the same order. That is, one can drop the second term in the brackets. Or, the power spectrum of the oscillating part in eqn (9) can be approximated by

$$
X\left(\Omega=\omega_{d}, \tau\right) \approx \frac{C_{1}}{\sqrt{2 \pi}} \cdot \frac{e^{\zeta \omega_{n}(\tau-L)}-e^{-\zeta \omega_{n}(\tau+L)}}{\zeta \omega_{n}} .
$$

This can be also observed from Figure 2 that the power is maximal when $\Omega \rightarrow \omega_{d}$. In addition, referring Figure 1 , the window length is set $2 \times L$. How-ever, the total length obviously needs at least one complete cycle to get the right transformed $\omega_{d}$. For this reason, $L \geq 2 \pi / \omega_{d}$ is used as the minimum length of window functions.

Taking the ratio of the two consecutive powers between $\tau$ and $(\tau+\Delta)$, which are evaluated right at $\omega_{d}$, together with the minimum length for the window, one obtains

$$
\left.\frac{X\left(\omega_{d}, \tau\right)}{X\left(\omega_{d}, \tau+\Delta\right)}\right|_{L=2 \pi / \omega_{d}} \approx e^{\zeta \omega_{n} \Delta}=\exp \left(\frac{c \Delta}{2 m}\right) .
$$

The upper neat form is readily derived and independent of constants $C_{1}$ and $C_{2}$, which indicate actually the two input energies, cf. Figure 2 for detail. In addition, the notations of the right most term in eqn (13) have been substituted by the system physical parameters. Notice that the center term of eqn (13) is the exact form of the inverse of the decaying exponent that appears in eqn (3). Therefore, eqn (13) provides additional information to identify the physical parameters $c$ and $m$, if the system damping is small such that $\omega_{n}$ can be reasonably replaced by $\omega_{d}$. And, it also provides a way to estimate the dam-ping ratio $\zeta$ of the system:

$$
\zeta \approx \frac{1}{\omega_{d} \Delta} \ln \left[\frac{X\left(\omega_{d}, \tau\right)}{X\left(\omega_{d}, \tau+\Delta\right)}\right] .
$$


It is also worthy to note that eqn (14) is neither dependent of the initial conditions, nor of the input loads. However, the general form of the approximate power spectrum, eqn (12), is function of $C_{1}$, or the initial conditions.

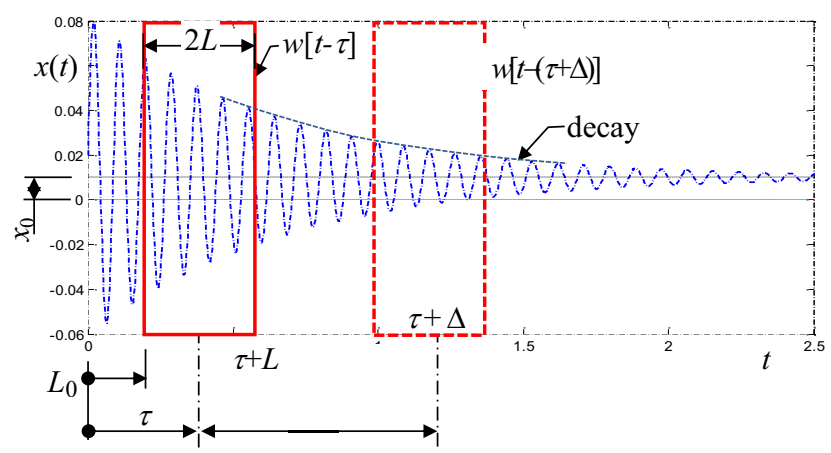

Figure 1. Concept of a moving window Fourier Transform.

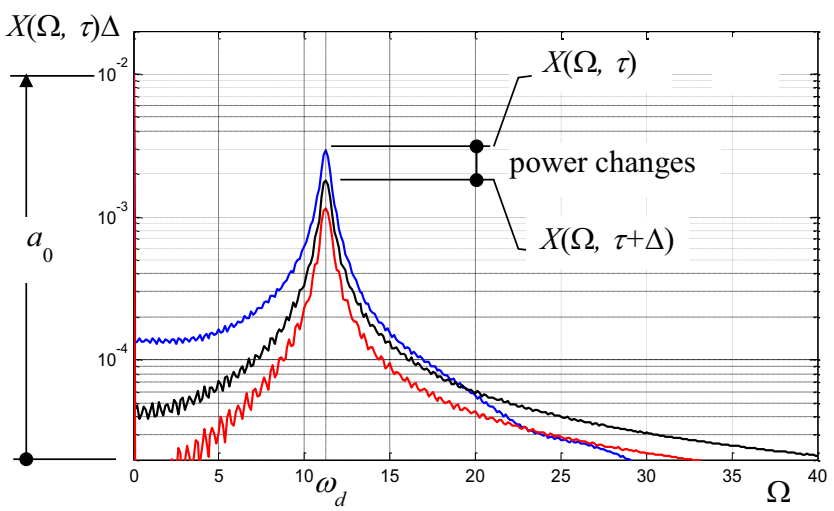

Figure 2. Response spectra of Figure 1 after transforming.

\subsection{System parameters}

Hence, taking the natural logarithms for the both sides of eqn (13), one can get the following relation equations

$$
\left\{\begin{array}{l}
\frac{c \Delta}{2 m}=\ln \left[\frac{X\left(\omega_{d}, \tau\right)}{X\left(\omega_{d}, \tau+\Delta\right)}\right]=a_{1} \\
\frac{k}{m} \approx \omega_{d}^{2}
\end{array}\right.
$$

where a short-hand notation $a_{1}$ has been adopted for the non-dimensional logarithmic value of this power spectra ratio. Using eqns (11) and (15), simultaneously solve the three system parameters, mass $m$, damping $c$ and stiffness $k$, the following expressions can be obtained

$$
\left\{\begin{array}{l}
k=\frac{F_{0}}{a_{0}}, \\
m=\frac{F_{0}}{a_{0} \cdot \omega_{d}^{2}}, \\
c=\frac{1}{\Delta} \frac{2 a_{1} \cdot F_{0}}{a_{0} \cdot \omega_{d}^{2}},
\end{array}\right.
$$

Therefore, in accordance with eqns (16), it is possible to estimate the parameters of sdof systems if the system is struck by a known external step excitation. Note that all parameters in the RHS of eqn (16) can be estimated from the signal power spectra. In addition, eqn (15a) can be further recast as

$$
a_{1}=\frac{1}{r} \ln \left[\frac{X\left(\omega_{d}, \tau\right)}{X\left(\omega_{d}, \tau+r \cdot \Delta\right)}\right],
$$

where $r$ is an integer. Or, it simply implies that $\Delta$ can be used as a parameter for tuning the sensitivity of identification.

\subsection{Summary for complete procedures}

Though the previous derivation has followed this procedure, complete procedures for applying the present method to determine the parameters of an sdof system is summarized as follows:

1) Apply a known external step excitation to the target structural system. Notice the system damping has to be small, for example $\zeta \leq 10 \%$.

2) To measure responses (displacement) at the locations of interest, says $x_{i}(t), i=1,2, \ldots, n$. The total length of the signals should be long enough so that the next analyses can be accurately completed.

3) To identify the damped natural frequencies, $\omega_{i}^{\prime}$, from the measured signals in the frequency domain. The averaged of these $n \omega_{i}^{\prime}$ will be taken as $\omega_{\mathrm{d}}$.

4) To apply mwFT to these $x_{i}(t)$ signals and get their corresponding spectra $X_{i}(\Omega, \tau+r \cdot \Delta)$ for all $r=1$, $2, \ldots, \ell$ for individual measurements. It is recommended to discard the first few oscillations for all $x_{i}(t)$.

5) To complete identifying the system parameters using eqns (16), starting with system stiffness $k$. Appropriate statistic measures are recommended.

\section{Numerical verifications}

Following the procedure of the last section, numerical studies are performed to verify the present algorithm. In order to compare the identified parameters with the true input ones which are known a priori, all signals are generated by applying the Simulink Toolbox of MATLAB. Through the whole simulations, the numerical signals are acquired at the sampling frequency $500 \mathrm{~Hz}$. Meanwhile, the shift and length of the moving windows, $\Delta$ and $L$, as well as the window function are all kept unchanged for all examples. The type of a hanning window is chosen since that it has been found [4] more robust and accurate. In addition, all these physical parameters are in consistent units and neglected hereafter.

\subsection{Model of a bridge crane}

The specification of the bridge crane which is taken as the example is listed in Table 1. In accordance with CAE results, the corresponding undamped natural frequency is $1.96 \mathrm{~Hz}$. If the crane is to suddenly lift a weight, $F_{0}$, when the hoister is at the center of the girder, then this loading 
can be regarded as a step load. Or, neglecting the reinforcement inside the two girders, the equivalent sdof system has the equation of motion in its deflection $y(t)$ :

$$
m \ddot{y}+c \dot{y}+k y=F_{0} \cdot u(t),
$$

with $m=35,000 \mathrm{~kg}, k=6,200 \mathrm{~N} / \mathrm{mm}$, and $c$ is set to 46 $\mathrm{N}-\mathrm{s} / \mathrm{mm}$ or the approx. damping ratio is ca. $5 \%$, which is believed practically reasonable. Figure 3 shows the 3D schematic diagram of the model. Notice also from Figure 3(c) where shows the corresponding mode shape.

Table 1. Specification of the bridge crane.

\begin{tabular}{|c|c|c|c|c|c|}
\hline Rating & Span & \multicolumn{3}{|c|}{ Speed } & Lifting \\
\cline { 3 - 5 } Load & $S$ & Hoisting & Transv. & Travel & $H$ \\
\hline $\mathrm{m}$. Ton & $\mathrm{m}$ & $(\mathrm{m} / \mathrm{min})$ & $(\mathrm{m} / \mathrm{min})$ & $(\mathrm{m} / \mathrm{min})$ & $(\mathrm{m})$ \\
\hline 51.6 & 33 & $40 / 80$ & 150 & 100 & 17.9 \\
\hline
\end{tabular}
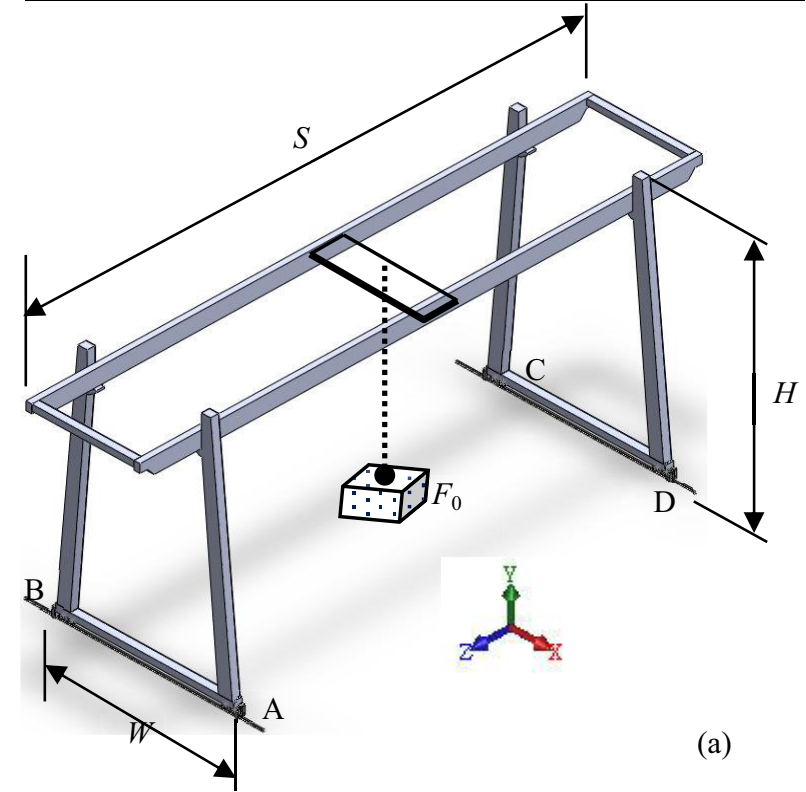

(a)

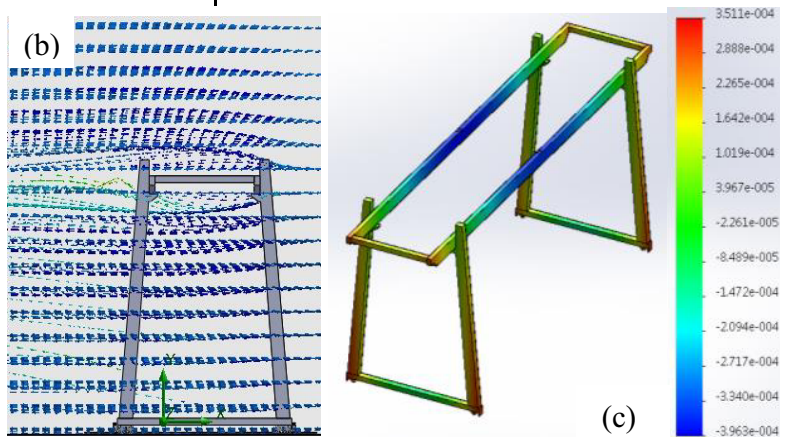

Figure 3. (a) A bridge crane and its sdof model; (b) the side view; and (c) $1^{\text {st }}$ mode shape in $y$-direction.

\subsection{Single-dof systems: parametric studies}

In addition to verify the validity of the present method, the authors intend to show how those given parameters affect to the final results. These parameters include: The window length $(L)$, the size of the step-load $\left(F_{0}\right)$, the discarded length of signal in the time domain $\left(L_{0}\right)$, and the shift $(\Delta)$ of window functions. All these parameters are in terms of non-dimensional quantities. That is, the lengths in the time domain are normalized by the period of the damped oscillation, and $F_{0}$ is divided by its weight of the system mass. Moreover, all errors are defined by

$$
\text { relative error }=\frac{(\text { estimated value }- \text { true value })}{\text { true value }} \text {. }
$$

\subsubsection{The effects of the window length $L$}

Firstly, one is interested in the choice of $L$ to the estimation of the system natural frequency, $f_{\mathrm{d}}$. Refer to Figure 4, it can be clearly seen if a reasonable accuracy is desired, one has to select the window length somewhat greater than 12 to 15 times of the period of the system oscillation. The plot also reveals that it always underestimates $f_{\mathrm{d}}$, or errors are in the negative side.

In addition to $f_{\mathrm{d}}$, the steady-state response is also a key factor since it is used as a quantity of calibrations. It is found that the accuracy of $a_{0}$ (estimated from the spectra) is quadratically deceasing with $L$ increases. However, to select $L \geq(12 \sim 15) / f_{d}$ can basically meet the engineering needs.

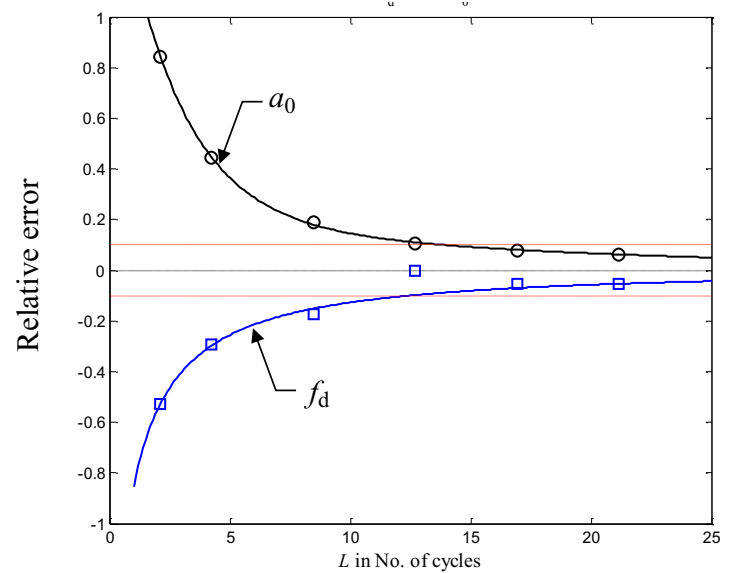

Figure 4. Effects of $L$ to the accuracy of $f_{\mathrm{d}}$ and $a_{0}$.

\subsubsection{The effects of the step-loads $F_{0}$}

The applying step-load, $F_{0}$, is to generate the signals of oscillations. It may cause lower SNR signals, if $F_{0}$ is too small. Figure 5 shows that it requires at least ca. $4 \% \sim 5 \%$ of its system mass to generate a good calibration quantity, $a_{0}$. Note also that a larger of $F_{0}$-to- $m g$ ratio tends to have better effects. On the other hand, however, it may not be practical in engineering practices. Refer to Figure 5 for detail.

\subsubsection{The effects of the discarded length, $L_{0}$}

It has been found that $L_{0}$ can be a factor which affecting the accuracy of identifying $a_{0}$. Figure 6 shows that the choice of $L_{0}$ to the accuracy of $a_{0}$. According this plot, at least ca. 3 first oscillating cycles of the signal after a stepload cannot be included to the present method. That is, depending on the initial conditions, users are not to include signals at least first $3 / f_{\mathrm{d}}$ to $4 / f_{\mathrm{d}}$ when signals are strongly influenced by those of initial conditions. 


\subsubsection{The effects of the window shift, $\Delta$}

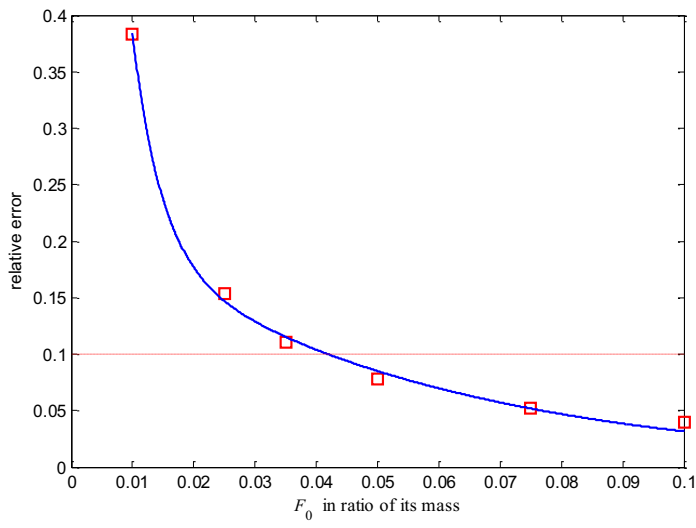

Figure 5. Effects of $F_{0}$ to the estimation of $a_{0}$.

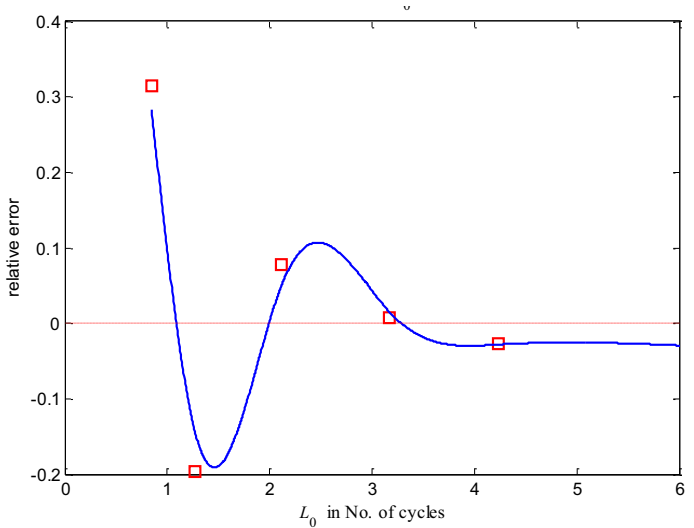

Figure 6. Effects of $L_{0}$ to the estimation of $a_{0}$.

Parameter $\Delta$ (the window shift) can be used as a tuning factor to adjust the sensitivity, according to eqn (13). Figure 7 displays the three system parameters vs. $\Delta$. The diagram implies that one is to choose $\Delta \geq(0.9 \sim 1.1) / f_{\mathrm{d}}$ so that the relative errors can be assured to minimum. Meanwhile, the trends of the three errors are quite similar.

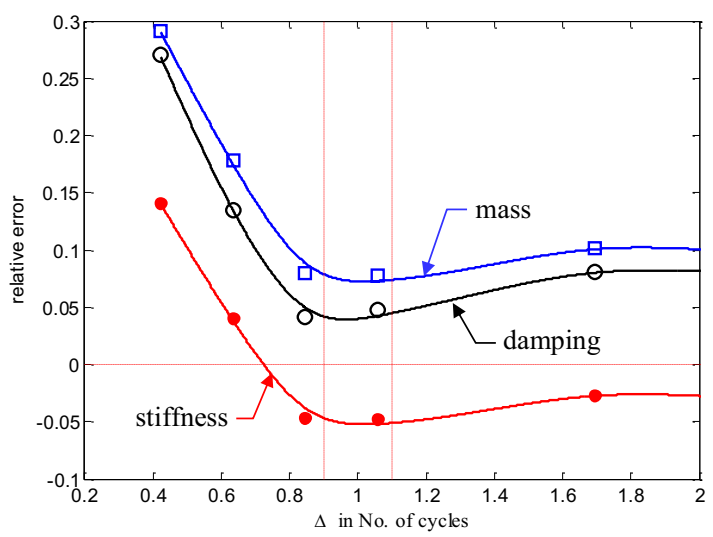

Figure 7. Effects of $\Delta$ to accuracies of the parameters.

\subsubsection{The effects of $\Delta$ and $L$}

The combined effects of window shift and its length $L$ to the estimation of $\zeta$ are shown in Fig. 8. Similar to those of in Fig. 7, one is able to have quite good estimates as long as to select $L$ is larger ca. 2 oscillations while $\Delta$ is somewhat larger than at least one oscillations.

\section{Concluding remarks}

A novel method that can be regarded as an "output-only" identification method has been proposed in the present report, though it requires a step-load as an external excitation force. The method is to measure both oscillating and the steady-state deflections after such step-loads. The next step is to apply the moving window Fourier transform to the former, while the latter is used as a calibration reference. The complete theoretical derivation as well as procedures has been well documented.

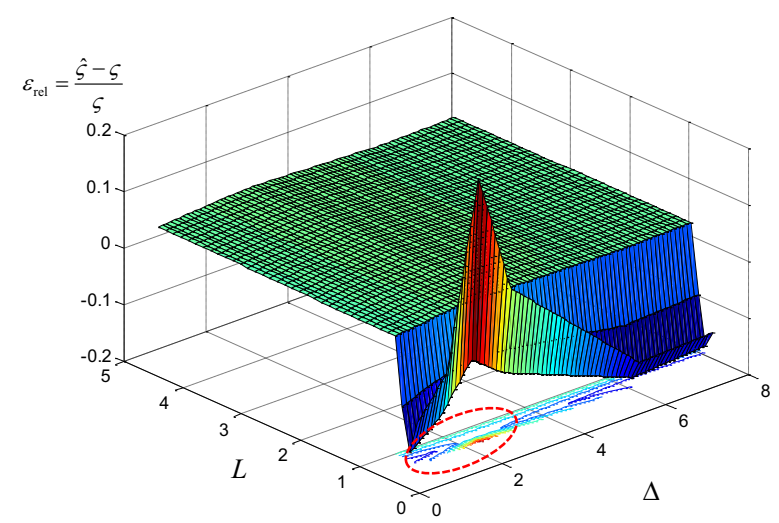

Figure 8. Effects of $\Delta$ and $L$ to accuracies of the parameters.

In addition, numerical verifications have been carried out to substantiate the validity of the new method. A bridge crane of single-dof model has been used as the example of such parametric studies. The four parameters, including the window length $(L)$ of the mwFT, the shift $(\Delta)$ of this window function, the size of the step-load $\left(F_{0}\right)$, and the discarded length of signal in the time domain $\left(L_{0}\right)$ are of the main concern. The simulation results show that the optimal parameter combinations for this new method are

$$
\left\{\begin{array}{l}
L \geq(12 \sim 15) / f_{d} \\
F_{0} \geq(4 \% \sim 5 \%) \times m g \\
\Delta \geq(1.0 \sim 1.1) / f_{d}
\end{array}\right.
$$

where $f_{\mathrm{d}}$ and $m$ are the system damped natural frequency in $\mathrm{Hz}$ and the mass, respectively. Furthermore, it is also recommended that to discard at least first 3 to 4 oscillations, which are violently oscillating, after the external step-load is applied. By doing so, the influence of impulsive part of the step-load to the steady state response can be alleviated. Nevertheless, the method can be very convenient to monitor structural system like cranes.

\section{References}

1. Z. Feng, M. Liang, F. Chu, Mech. Syst. Signal Pr. 38, 165 (2013)

2. W. Li, J. Sound Vib. 279, 1181 (2005)

3. Feeny BF., J Vib Acoust ASME 131, 041005-1 (2009)

4. R.Y. Hong, Master Thesis (in Chinese), Nat'l Taipei Univ. Tech (2012)

5. W. Li, K.-C. Liu, Y.-R. Lin, Int'l Conf. of Adv. Manuf., ICAM (2016) 
6. D.J. Inman, Engineering Vibration, $3^{\text {rd }}$ Ed., Pearson Edu., (2007)

7. A. Palamides and A. Veloni, Signals and Systems Laboratory with MATLAB, CRC Press, (2011) 